\title{
BMJ Open Measurement of visceral fat and abdominal obesity by single-frequency bioelectrical impedance and CT: a cross-sectional study
}

\author{
Zhengyang Xu, ${ }^{1,2}$ Yandong Liu (D) , ${ }^{3}$ Chengxi Yan, ${ }^{3}$ Ruopei Yang, ${ }^{3}$ Li Xu, ${ }^{3}$ Zhe Guo, ${ }^{3}$ \\ Aihong Yu, ${ }^{3}$ Xiaoguang Cheng (i) ${ }^{3}$ Lin Ma, ${ }^{2}$ Chunhong Hu, ${ }^{1,4}$ \\ Giuseppe Guglielmi, ${ }^{5,6}$ Karen Hind ${ }^{7}$
}

To cite: Xu Z, Liu Y, Yan C, et al. Measurement of visceral fat and abdominal obesity by single-frequency bioelectrical impedance and CT: a cross-sectional study. BMJ Open 2021;11:e048221. doi:10.1136/ bmjopen-2020-048221

- Prepublication history for this paper is available online. To view these files, please visit the journal online (http://dx.doi. org/10.1136/bmjopen-2020048221).

Received 22 December 2020 Accepted 03 August 2021

\section{ABSTRACT}

Objectives The measurement of visceral fat (VF) is clinically important for the identification of individuals at high risk of visceral obesity-related health conditions. Bioelectrical impedance analysis (BIA) is a widely available and frequently used body composition assessment method, but there have been few validation studies for the measurement of VF. This validation study investigated agreement between BIA and CT for the assessment of VF in adults.

Design Cross-sectional study.

Setting Between 2015 and 2016 in China.

Participants A total of 414 adults (119 men and 295 women) aged 40-82 years.

Primary and secondary outcome measures CT-visceral fat area (VFA) was derived at the L2-3 and umbilicus level and VFA cut-offs for visceral obesity applied. BIA measurements of visceral fat level were compared with CT VFA findings using scatter plots and receiver operator characteristic (ROC) curves.

Results Scatter plots showed poor agreement between BIA and CT-derived visceral fat measurements in both sexes $(R=0.387-0.636)$. ROC curves gave optimum figures for sensitivity and specificity of $65 \%$ and $69 \%$ in women and $76 \%$ and $70 \%$ in men, respectively, for BIA to discriminate between adults with normal levels of VF and those with visceral obesity determined by CT.

Conclusion BIA has limited accuracy for the assessment of VF in adults in practice when compared with the criterion method.

\section{INTRODUCTION}

An excess of visceral adipose tissue (VAT) can cause metabolic abnormalities, through the secretion of harmful inflammatory adipokines such as interleukin-6, tumour necrosis factoralpha and macrophage chemoattractant protein-1. ${ }^{1}$ In particular, visceral fat increases the risk for development of chronic low-grade inflammation and is involved in the pathogenesis of numerous inflammatory medical conditions including metabolic syndrome, diabetes and cardiovascular disease, ${ }^{2-4}$ as well
Strengths and limitations of this study

- The agreement of bioelectrical impedance analysis (BIA) with CT for the assessment of visceral fat and abdominal obesity in adults was poor.

- We found improved visceral fat level thresholds in men and women compared to the manufacturer's recommendation.

- In this study, the BIA device was single frequency and therefore findings cannot be generalised to multifrequency BIA devices.

as being an important, independent predictor of all-cause mortality. ${ }^{45}$ It is therefore clinically important to identify individuals with high levels of visceral fat, so that appropriate interventions can implemented.

Proxy measures of excess fat accumulation such as body mass index (BMI) and waist circumference have been demonstrated to be largely ineffective in identifying visceral obesity, although waist-to-height ratio has shown promise. ${ }^{67}$ The gold standard methods for the measurement of visceral fat are CT and MRI. Visceral fat area (VFA) based on single-slice imaging of CT/MRI is widely used in research studies ${ }^{89}$ but rarely used in clinical practice. Several studies have provided cut-off values of VFA for visceral obesity assessment in Japanese, Korean and Chinese populations, ${ }^{10-12}$ recognising a greater amount of visceral adiposity at any given BMI in East Asian populations compared with other ethnic groups like white population, African Caribbean black population and Hispanics. ${ }^{13}$ However, CT and MRI are limited in largescale studies or in clinical protocols, due to cost, availability and radiation exposure.

Bioelectrical impedance analysis (BIA) is a widely available, low-cost and non-X-ray-based method, and is used frequently in clinical 
practice and research settings to evaluate total body water and body composition. There have been few validation studies of BIA-derived assessments of visceral fat, ${ }^{14}$ and no study has yet investigated BIA-estimates in accord with CT-derived visceral obesity reference cut-points. Therefore, the aim of this study was to investigate agreement between single-frequency BIA and abdominal CT for the assessment of visceral fat and visceral obesity in Chinese adults.

\section{MATERIALS AND METHODS Study participants}

Participants were recruited from community-based population samples of the Changzhou region from the Prospective Urban Rural Epidemiology China Action on Spine and Hip status study. ${ }^{16}$ The inclusion criterion and exclusion criterion have been described previously. ${ }^{16}$ In addition, for this study, individuals who had hydration abnormalities such as visible oedema, cirrhosis or heart failure were excluded from the study. The overall study population included 414 adults, comprising 119 males (age range: 40-82 years) and 295 females (age range: 44-81 years).

\section{Anthropometry}

Anthropometry, BIA and CT assessments were performed during the same appointment for each participant. Height and weight were measured using calibrated equipment (HW-900Y, Lejia Tech Corp) with participants wearing underwear and barefoot. BMI was calculated as weight $(\mathrm{kg})$ divided by height squared (metre). A BMI of $\leq 24 \mathrm{~kg} / \mathrm{m}^{2}$ was considered normal weight and $>24 \mathrm{~kg} / \mathrm{m}^{2}$ was defined as overweight/obesity. ${ }^{17}$

\section{Quantitative CT abdominal fat area}

Abdominal CT scans were performed on a Siemens Somatom Definition AS+64 slice dual energy CT scanner. The scanning range was from superior margin level of T12 vertebral body to L5 inferior margin. Scanning parameters were $120 \mathrm{KV}, 125 \mathrm{mAs}, 500 \mathrm{~mm}$ field of view, 1-mm slice thickness, matrix $512 \times 512$, table height $154 \mathrm{~cm}$. All participants were positioned supine on the CT table, and a quantitative CT (QCT) calibration phantom (Mindways, Austin, TX, USA) was placed beneath the body. All original DICOM images were transferred to QCT analysis software (QCT PRO 5.0) workstation in Beijing Jishuitan Hospital and converted into QCT documents. The tissue composition module was used to measure total fat area (TFA) and VFA at L2-L3 intervertebral space (figure 1A,B) and umbilicus cross-section level. Details of adipose tissue measurements have been reported previously. ${ }^{18}$ In brief, adipose tissue was segmented and mapped in blue with a default threshold, and the outer contour of abdominal wall was then outlined by the software automatically on each $1 \mathrm{~mm}$-thick slice. All measurements were carried out by two trained and experienced radiologists (CY and RY). The interobserver and intraobserver reliabilities of QCT
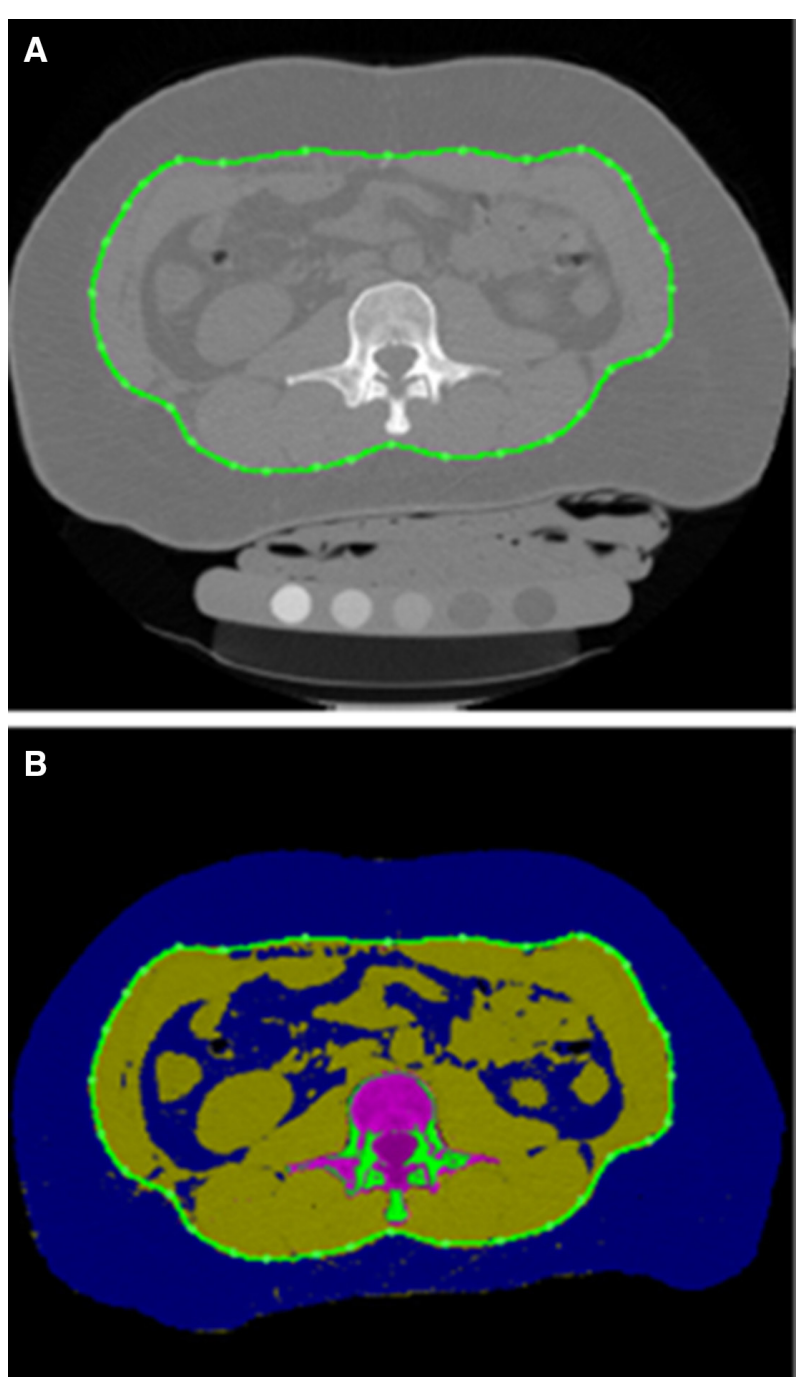

Figure 1 (A) Quantitative CT fat image of the abdomen. (B) Segmentation results in the fat map: abdomen fat is shown in blue colour and visceral adipose tissue is shown by the green contour.

VFA measurements were good with intraclass correlation coefficient 0.996 and $0.990,{ }^{8}$ respectively.

\section{BIA body composition}

Body composition was estimated using whole-body, upright, single-frequency (SF)-BIA (Tanita BC-554, Tanita Corp, Tokyo, Japan). All participants were measured in lightweight clothing and standing barefoot on the metal footpads. To measure the bio-impedance, a very low, safe electrical signal is sent from four metal electrodes through the feet to the legs and abdomen. The Tanita BIA uses a SF-BIA at $50 \mathrm{kHz}$ which predominately measures extracellular water and approximately $25 \%$ of intracellular water. Participant information entered into the system to enable the computing of the BIA algorithms, included gender, age, height and weight. Body fat mass percentage (BF) and visceral fat level (VFL) were recorded as the mean value of two repeated measurements. The time interval between the BIA and QCT measurements did not exceed 7 days. The Tanita body composition analyser gave a 
Table 1 Characteristics of the study population

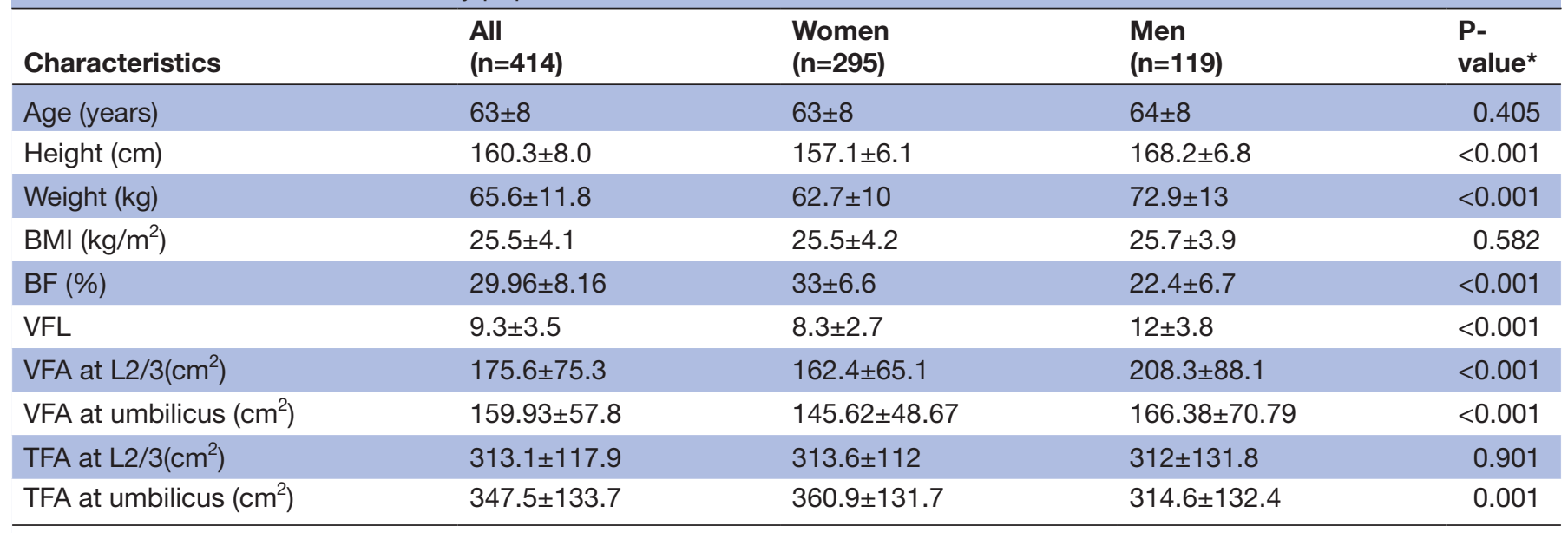

Data are expressed as mean \pm SD.

${ }^{*} \mathrm{P}$ for difference between gender groups.

BF, body fat percent; BMI, body mass index; TFA, total fat area; VFA, visceral fat area; VFL, visceral fat level.

range of VFL rating between 1 and 59. According to the manufacturer's information, a rating between 1 and 12 indicates a healthy level of visceral fat, whereas a rating between 13 and 59 indicates excess visceral fat. The reproducibility of estimated values using this BIA system have been reported previously. ${ }^{18} 19$

\section{Statistical analysis}

Statistical analyses were performed using SPSS V.25.0 software (IBM, Armonk, NY, USA) and R V.3.6.2 (R Core Team, R Foundation for Statistical Computing, Vienna, Austria). The measurement data are presented as the mean \pm SD. The Mann-Whitney U test was used for intergroup and subgroup comparisons of baseline characteristics. Spearman's rank correlation coefficients were used to evaluate whether VFL was correlated with other parameters. Pearson correlation coefficients were determined among the anthropometric parameters, body fat variables measured by CT and BF. A correlogram was used to plot a graph of correlation matrix. In this plot, correlation coefficients were coloured and sized according to the value. Statistical analyses were performed to assess the prevalence of visceral obesity based on BIA VFL $($ VFL $>13)$ and VFA (VFA $>142 \mathrm{~cm}^{2}$ for men and $115 \mathrm{~cm}^{2}$ for women at L2/3 level; VFA $>111 \mathrm{~cm}^{2}$ for men and $96 \mathrm{~cm}^{2}$ for women at the umbilical level) ${ }^{12}$ by CT. Scatter plots of VFL against VFA were drawn and receiver operator characteristic (ROC) curves used to determine the sensitivity and specificity for BIA measurements to discriminate between adults with normal levels of visceral fat and those with visceral obesity determined by CT. $p<0.05$ was considered statistically significant.
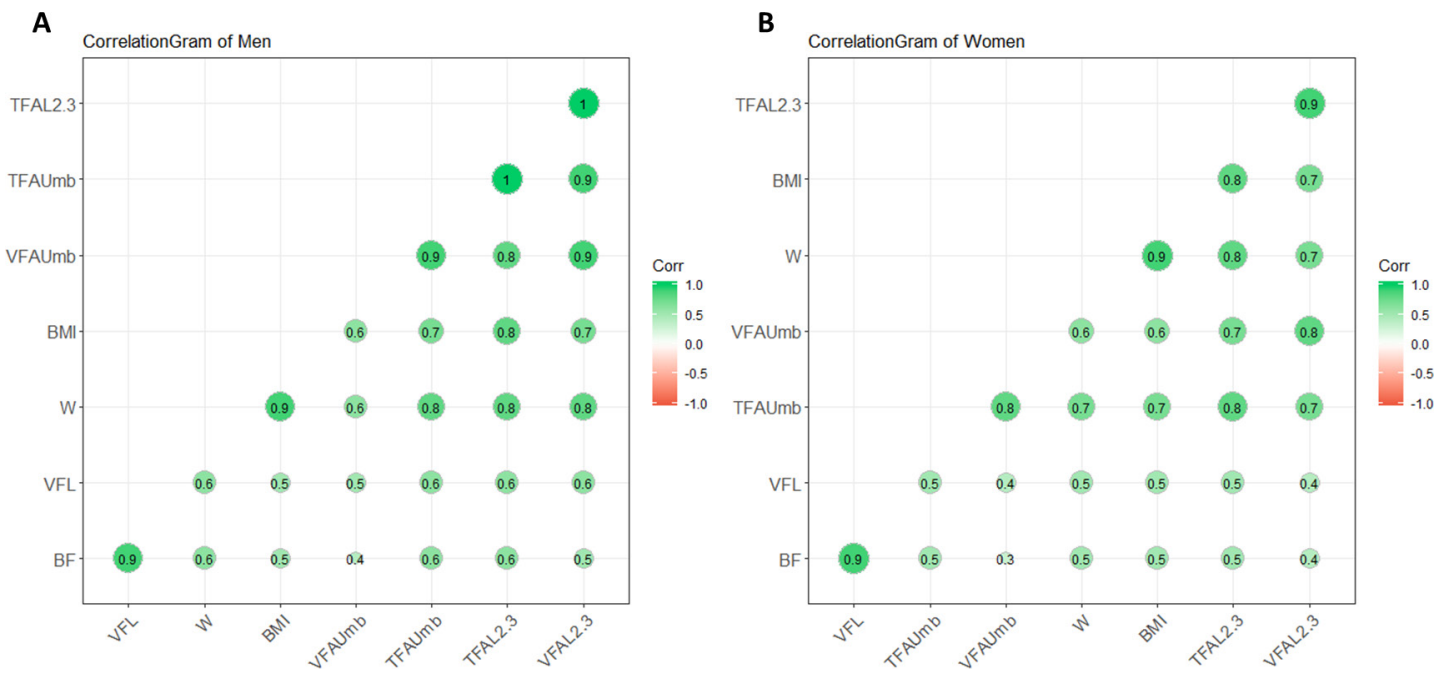

Figure 2 Correlation matrix diagram of fat variables, weight and BMl in (A) men and (B) women. Correlation coefficients are coloured and sized according to the value. Note: the correlation coefficients between TFA L2.3 and VFA L2.3 in (A), as well as TFA L2.3 and TFA Umb, are not 1 but 0.951 and 0.958 , respectively. All correlations were significant $(p<0.001)$. bf, body fat percent; BMI, body mass index; L2.3, lumber 2/3 level; TFA, total fat area; Umb, umbilicus; VFA, visceral fat area; VFL, visceral fat level; W, weight. 
Table 2 Characteristics of the study population stratified by BMI

\begin{tabular}{|c|c|c|c|c|c|c|}
\hline \multirow[b]{2}{*}{ Characteristics } & \multicolumn{2}{|l|}{ Women } & \multirow[b]{2}{*}{$P$ value } & \multicolumn{2}{|l|}{ Men } & \multirow[b]{2}{*}{$P$ value } \\
\hline & Non-obesity $(n=216)$ & Obesity (n=79) & & $\begin{array}{l}\text { Non-obesity } \\
(n=139)\end{array}$ & Obesity (n=73) & \\
\hline $\mathrm{BF}(\%)$ & $31.6 \pm 5.8$ & $36.8 \pm 6.9$ & $<0.001$ & $21 \pm 5.9$ & $26.6 \pm 7.2$ & $<0.001$ \\
\hline VFL & $7.5 \pm 2.1$ & $10.3 \pm 3.2$ & $<0.001$ & $11.2 \pm 3.3$ & $14.4 \pm 4.1$ & $<0.001$ \\
\hline TFA at $\mathrm{L} 2 / 3\left(\mathrm{~cm}^{2}\right)$ & $270.5 \pm 84.5$ & $431.3 \pm 91.6$ & $<0.001$ & $269.4 \pm 102.1$ & $444 \pm 127.5$ & $<0.001$ \\
\hline TFA at umbilicus $\left(\mathrm{cm}^{2}\right)$ & $317.3 \pm 106.4$ & $479.8 \pm 122$ & $<0.001$ & $274.3 \pm 100.2$ & $439.4 \pm 145.7$ & $<0.001$ \\
\hline
\end{tabular}

A BMI of $\leq 28 \mathrm{~kg} / \mathrm{m}^{2}$ was considered non-obesity and $>28 \mathrm{~kg} / \mathrm{m}^{2}$ was defined as obesity.

$\mathrm{BF}$, body fat percent; BMI, body mass index; TFA, total fat area; VFA, visceral fat area; VFL, visceral fat level.

\section{Patients and public involvement}

Patients and the public were not involved in this study, including data collection, analysis and interpretation.

\section{RESULTS}

Anthropometric, body fat percentage and visceral fat parameters are shown in table 1 . There were significant differences in height, weight, BMI, body fat percentage, VFL and VFA between women and men.

Figure 2 shows the plots of correlation matrix of body fat composition variables and anthropometric measurements in men (figure 2A) and women (figure 2B). VFL was poorly correlated with VFA and TFA at L2/3 and umbilicus level $(\mathrm{R}=0.387-0.636$, all $\mathrm{p}<0.001)$ in both genders. The correlation between VFL and BF was good in both sexes ( $\mathrm{R}=0.851$ for women and 0.894 for men, $\mathrm{p}<0.001)$. BMI and weight showed higher associations $(\mathrm{R}=0.586-0.762$, all $\mathrm{p}<0.001)$ with VFA than VFL $(\mathrm{R}=0.384-0.565$, all $\mathrm{p}<0.001)$. Total body fat percentage was poorly associated with VFA and TFA at both levels $(\mathrm{R}=0.335-0.506$, all $\mathrm{p}<0.001)$.

Table 2 shows BIA and CT-derived fat mass results for normal weight and overweight/obesity subgroups. Significant differences $(p<0.001)$ were found between overweight/obesity and normal weight subgroups for all body fat composition parameters in both sexes (table 2). Figures 3 and 4 demonstrate the level of agreement between BIA and CT for the identification of visceral obesity in women and men, stratified by BMI, respectively. Approximately $10 \%$ of overweight/obese women and no normal-weight women were correctly identified as having high levels of visceral fat by BIA. Conversely, CT imaging identified high levels of visceral fat in $40 \%$ of normalweight women. In overweight/obese men, the agreement between BIA and CT was slightly better, with BIA correctly identifying $50 \%$ of men with visceral obesity in the overweight/obese group. While in normal-weight men, BIA only correctly identified $5 \%$ of men with visceral obesity.
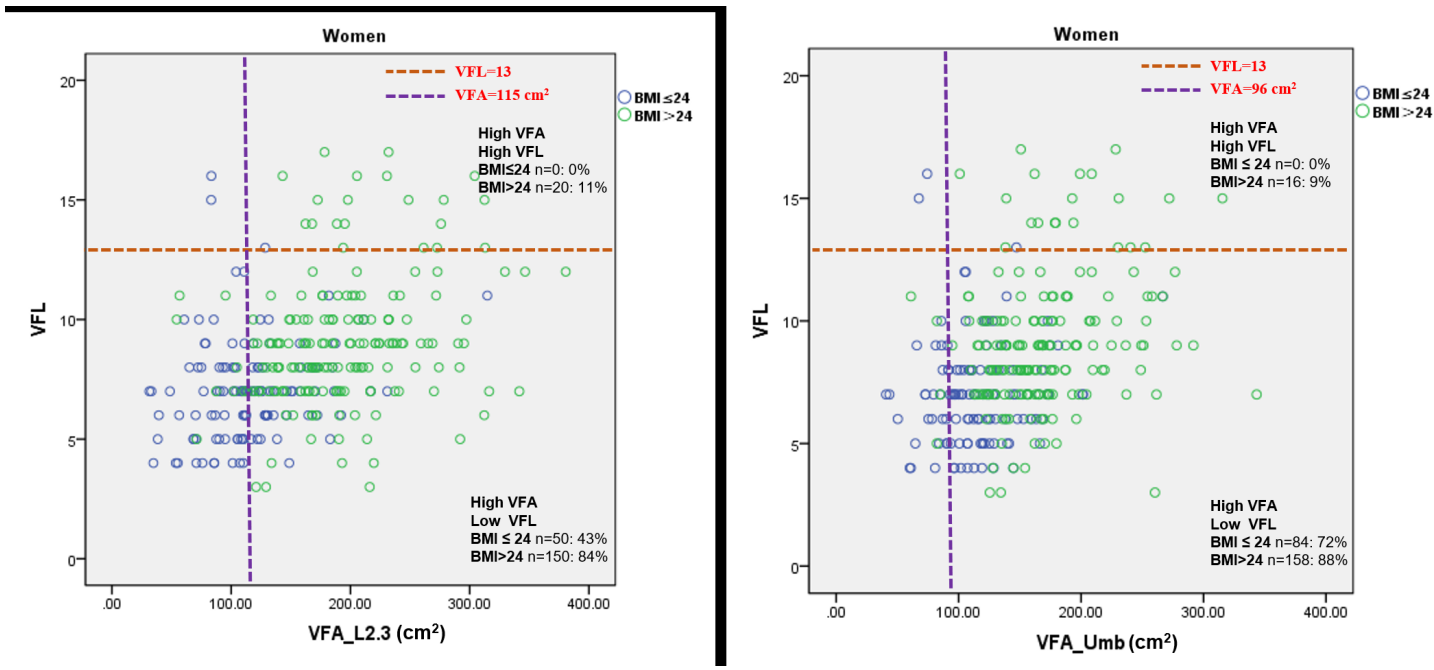

Figure 3 Plots of VFL and VFA at L2/3 and umbilicus levels in women. Visceral obesity: visceral fat area determined by CT $>115 \mathrm{~cm}^{2}$ at $\mathrm{L} 2 / 3$ and $>96 \mathrm{~cm}^{2}$ at umbilicus level. Percentage was defined as number of subjects in the quadrant/number of subjects in related BMI group. BMI, body mass index; L2.3, lumber 2/3 level; Umb, umbilicus; VFA, visceral fat area; VFL, visceral fat level. 

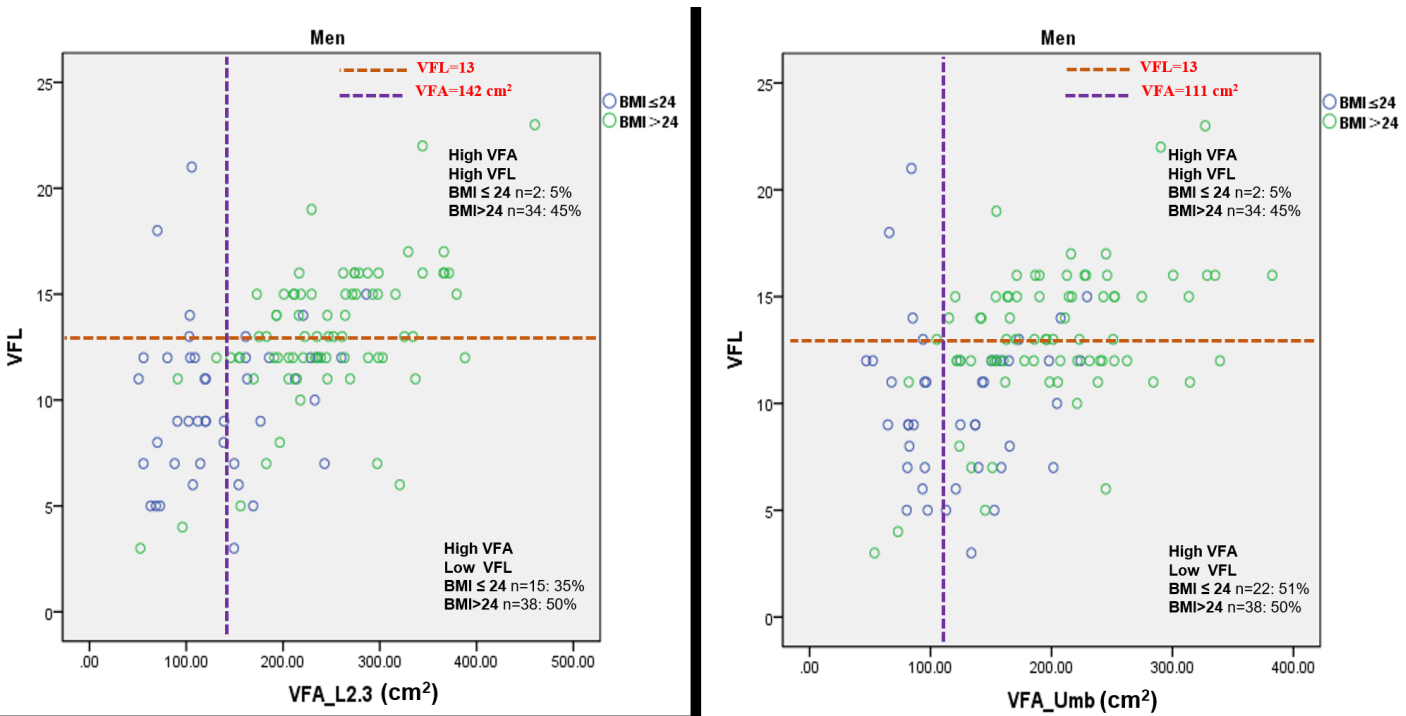

Figure 4 Plots of VFL and VFA at L2/3 and umbilicus levels in men. Visceral obesity: visceral fat area determined by CT $>142 \mathrm{~cm}^{2}$ at L2/3 and $>111 \mathrm{~cm}^{2}$ at umbilicus level. Percentage was defined as number of subjects in the quadrant/number of subjects in the related BMI group. BMI, body mass index; L2.3, lumber 2/3 level; Umb, umbilicus; VFA, visceral fat area; VFL, visceral fat level.

Figures 5 and 6 show the corresponding ROC curves. A BIA VFL threshold of 8 gave $65 \%$ sensitivity and $69 \%$ specificity for identifying women with VAT $>115 \mathrm{~cm}^{2}$ at L2/3. A BIA threshold of 12 gave $76 \%$ sensitivity and $70 \%$ specificity for identifying men with VAT $>142 \mathrm{~cm}^{2}$ at L2/3. Overall there was poor agreement between the two methods for the assessment of visceral obesity.

\section{DISCUSSION}

Abdominal adipose tissue can be measured accurately using the state-of-art imaging techniques such as CT. However, due to increased ionising radiation and highcost, CT is inappropriate for the measurement and monitoring of abdominal visceral fat in many research and clinical situations. As such, BIA, as a more widely available and low-cost body composition tool is more feasible, at least in clinical practice. However, we found poor agreement between BIA and CT for the measurement of visceral fat. The correlation coefficients ( $\mathrm{R}=0.387-0.636)$ for visceral fat between BIA and CT in this study (figure 2A,B) are similar to those reported elsewhere between BIA and MRI $\left(r^{2}=0.13-0.44\right) .{ }^{15}$ At the manufacturer's recommended VFL threshold of 13 the sensitivity and specificity of BIA measurements to discriminate visceral obesity measured by CT VFA were $10 \%$ and $97 \%$, respectively, in women and $52 \%$ and $90 \%$ in men. However, we found improved figures for sensitivity and specificity by choosing different VFL thresholds in men and women.

Another study using two whole-body BIA devices and one abdominal BIA device found that agreement between
A

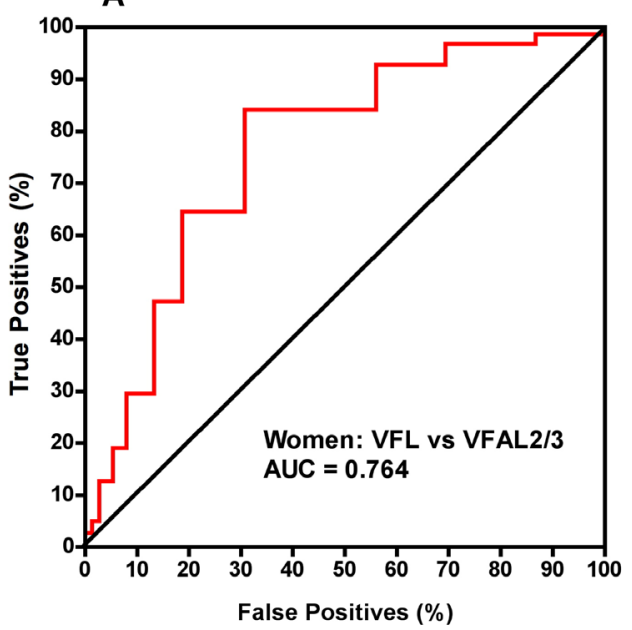

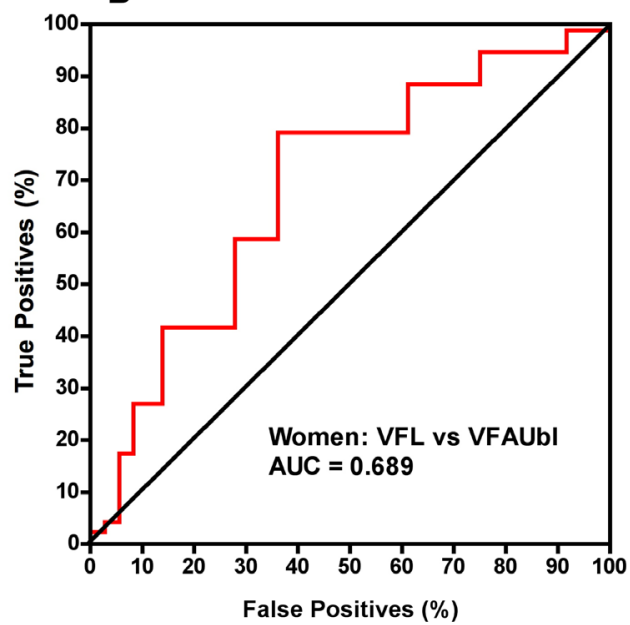

Figure 5 ROC curves for the plots of VFL and VFA at L2/3 and umbilicus levels in women in figure 3. Positive and negative cases of obesity in women were defined by reference to the VFA thresholds in figure 3. L2/3, lumber $2 / 3$ level; ROC, receiver operator characteristic; VFA, visceral fat area; VFL, visceral fat level. 
A

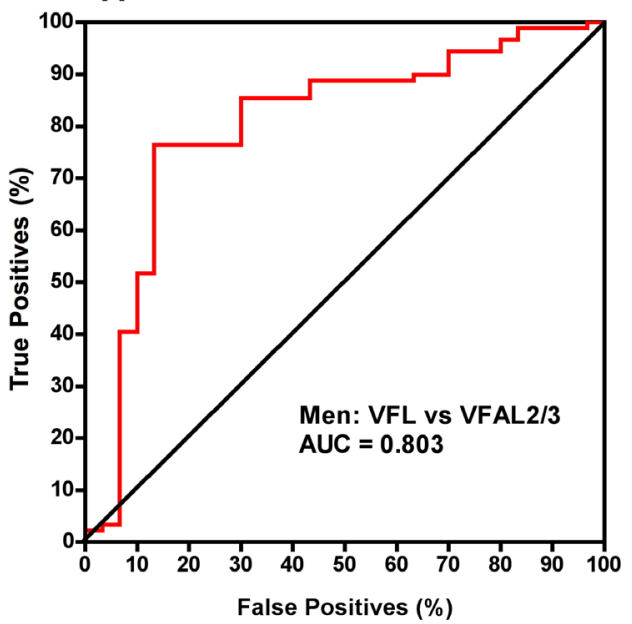

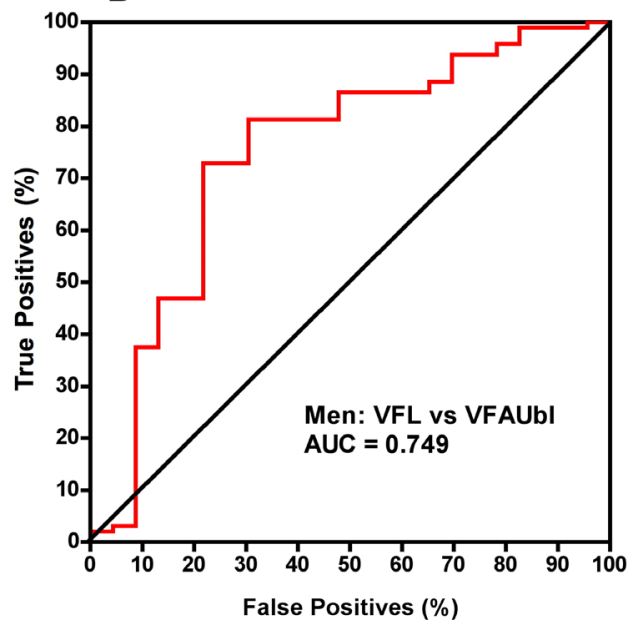

Figure 6 ROC curves for the plots of VFL and VFA at L2/3 and umbilicus levels in men in figure 4. Positive and negative cases of obesity in men were defined by reference to the VFA thresholds in figure 4. L2/3, lumber 2/3 level; ROC, receiver operator characteristic; VFA, visceral fat area; VFL, visceral fat level

all three BIA devices for visceral fat assessment was better for total fat mass than for visceral fat in both men and women. ${ }^{20}$ There has been some recent interest in the potential of locally applied BIA for the quantification of abdominal subcutaneous fat thickness. ${ }^{21}$ In addition, two previous studies have reported positive correlations between BIA-derived visceral fat measures and metabolic parameters including blood pressure, lipid profiles and fasting glucose. ${ }^{22}{ }^{23}$ However, it should be considered that the correlation coefficients for visceral fat and metabolic parameters in these studies are relatively low $(\mathrm{R}=0.2-0.4)$ and interestingly, the correlation coefficients were better for waist circumference. ${ }^{22} 23$

Unlike CT, BIA does not provide a direct measure of fat tissue. ${ }^{24}$ BIA most closely estimates body water and there is no direct theoretical relationship between resistance and/or reactance and relative body fatness. ${ }^{25}$ The estimation of adiposity from BIA is instead based on empirical relationships from samples of experimental subjects and calculations involve assumptions at several steps. ${ }^{26}$ Given the uncertainties surrounding the BIA-VFL calculation process, the significant disagreement between BIA and CT for defining visceral obesity requires further exploration.

We recognise several limitations to this study. First, it should be considered that we did not include measurements of waist circumference or waist-to-height ratio, of which the latter has been found to be highly correlated with visceral fat mass using dual-energy X-ray absorptiometry. ${ }^{7} \mathrm{BMI}$ is used to assess general obesity, while waist circumference is used to assess abdominal obesity. Therefore, it might be better to do the analyses of figures 3 and 4 stratified by waist circumference rather than BMI. Second, in this study, the BIA device was single frequency and therefore findings cannot be generalised to multifrequency BIA devices. Notably, different types of BIA equipment on the market include SF and multifrequency devices, which vary in price. The instrument (Tanita
BC-554) used in this study is a consumer-grade instrument and relatively inexpensive (US\$170) compared with professional-grade instruments (>US\$1000). It is important to acknowledge the wide range of variability in the accuracy of BIA scales and the comparative validities of SF and multifrequency BIA devices has also been questioned. ${ }^{26}$

\section{CONCLUSION}

The agreement of BIA with the criterion method, CT, for the assessment of visceral fat and abdominal obesity in adults was poor. Further studies are warranted to improve the predictive value of abdominal BIA relative to the gold standard of CT/MRI, before BIA should be accepted for the definition of visceral obesity in practice.

\section{Author affiliations}

${ }^{1}$ Department of Radiology, the First Affiliated Hospital of Soochow University, Suzhou, Jiangsu, China

${ }^{2}$ Department of Radiology, the First Medical Center of Chinese PLA General Hospital, Beijing, Beijing, China

${ }^{3}$ Department of Radiology, Beijing Jishuitan Hospital and Fourth Medical College of Peking University, Beijing, China

${ }^{4}$ Institute of Medical Imaging, Soochow University, Suzhou, Jiangsu, China ${ }^{5}$ Department of Clinical and Experimental Medicine, Foggia University School of Medicine, University Campus of Barletta, Dimiccoli Hospital, Foggia, Italy

${ }^{6}$ Department of Radiology, University of Foggia, Foggia, Italy

${ }^{7}$ Department of Sport and Exercise Sciences, Durham University, Durham, UK

Contributors Study design: $\mathrm{CH}, \mathrm{ZX}$ and $\mathrm{XC}$; study conduct: $\mathrm{CH}$ and $\mathrm{ZX}$; data collection: $X C, Y L, C Y, R Y$ and $L M$; data analysis: $Y L$ and $L X$; data interpretation: ZG and $\mathrm{AY}$; manuscript drafting: $\mathrm{CH}, \mathrm{ZX}, \mathrm{YL}, \mathrm{XC}, \mathrm{GG}$ and $\mathrm{KH}$; approving final version of manuscript: all authors.

Funding This study was supported by the National Natural Science Foundation of China (grant no. 81771831);the Beijing Natural Science Foundation project (grant number: 17L20188) and the Beijing Municipal Administration of Hospitals Clinical Medicine Development of Special Funding Support (code: XMLX201843).

Competing interests None declared.

Patient and public involvement Patients and/or the public were not involved in the design, or conduct, or reporting, or dissemination plans of this research. 
Patient consent for publication Not applicable.

Ethics approval This study was approved by the Research Ethics Committee of Beijing Jishuitan Hospital, and written informed consent was provided by all participants (approval number no. 201512-02).

Provenance and peer review Not commissioned; externally peer reviewed.

Data availability statement The datasets generated and/or analyzed during the current study are available from the Prof. Xiaoguang Cheng on reasonable request.

Open access This is an open access article distributed in accordance with the Creative Commons Attribution Non Commercial (CC BY-NC 4.0) license, which permits others to distribute, remix, adapt, build upon this work non-commercially, and license their derivative works on different terms, provided the original work is properly cited, appropriate credit is given, any changes made indicated, and the use is non-commercial. See: http://creativecommons.org/licenses/by-nc/4.0/.

\section{ORCID iDs}

Yandong Liu http://orcid.org/0000-0002-4752-9484

Xiaoguang Cheng http://orcid.org/0000-0002-4596-8248

\section{REFERENCES}

1 Fontana L, Eagon JC, Trujillo ME, et al. Visceral fat adipokine secretion is associated with systemic inflammation in obese humans. Diabetes 2007:56:1010-3.

2 Després J-P, Lemieux I, Bergeron J, et al. Abdominal obesity and the metabolic syndrome: contribution to global cardiometabolic risk. Arterioscler Thromb Vasc Biol 2008;28:1039-49.

3 Gruzdeva O, Borodkina D, Uchasova E, et al. Localization of fat depots and cardiovascular risk. Lipids Health Dis 2018;17:218.

4 Britton KA, Massaro JM, Murabito JM, et al. Body fat distribution, incident cardiovascular disease, cancer, and all-cause mortality. $J$ Am Coll Cardiol 2013;62:921-5.

5 Kuk JL, Katzmarzyk PT, Nichaman MZ, et al. Visceral fat is an independent predictor of all-cause mortality in men. Obesity 2006;14:336-41.

6 Camhi SM, Bray GA, Bouchard C, et al. The relationship of waist circumference and BMI to visceral, subcutaneous, and total body fat: sex and race differences. Obesity 2011;19:402-8.

7 Swainson MG, Batterham AM, Tsakirides C, et al. Prediction of whole-body fat percentage and visceral adipose tissue mass from five anthropometric variables. PLoS One 2017;12:e0177175.

8 Cheng $X$, Zhang Y, Wang C, et al. The optimal anatomic site for a single slice to estimate the total volume of visceral adipose tissue by using the quantitative computed tomography (QCT) in Chinese population. Eur J Clin Nutr 2018;72:1567-75.

9 Shen W, Punyanitya M, Wang Z, et al. Total body skeletal muscle and adipose tissue volumes: estimation from a single abdominal crosssectional image. J Appl Physiol 2004;97:2333-8.

10 Examination Committee of Criteria for 'Obesity Disease' in Japan, Japan Society for the Study of Obesity. New criteria for 'obesity disease' in Japan. Circ J 2002;66:987-92.
11 Lee A, Kim YJ, Oh S-W, et al. Cut-Off values for visceral fat area identifying Korean adults at risk for metabolic syndrome. Korean $J$ Fam Med 2018;39:239-46.

12 Huo L, Li K, Deng W, et al. Optimal cut-points of visceral adipose tissue areas for cardiometabolic risk factors in a Chinese population: a cross-sectional study. Diabet Med 2019;36:1268-75.

13 Nazare J-A, Smith JD, Borel A-L, et al. Ethnic influences on the relations between abdominal subcutaneous and visceral adiposity, liver fat, and cardiometabolic risk profile: the International study of prediction of intra-abdominal adiposity and its relationship with cardiometabolic Risk/Intra-Abdominal adiposity. Am J Clin Nutr 2012;96:714-26.

14 Browning LM, Mugridge O, Chatfield MD, et al. Validity of a new abdominal bioelectrical impedance device to measure abdominal and visceral fat: comparison with MRI. Obesity 2010;18:2385-91.

15 Chaudry O, Grimm A, Friedberger A, et al. Magnetic resonance imaging and bioelectrical impedance analysis to assess visceral and abdominal adipose tissue. Obesity 2020;28:277-83.

16 Li K, Zhang Y, Wang L, et al. The protocol for the prospective urban rural epidemiology China action on spine and hip status study. Quant Imaging Med Surg 2018;8:667-72.

17 Zhou B-F, Cooperative Meta-Analysis Group of the Working Group on Obesity in China. Predictive values of body mass index and waist circumference for risk factors of certain related diseases in Chinese adults--study on optimal cut-off points of body mass index and waist circumference in Chinese adults. Biomed Environ Sci 2002;15:83-96.

18 Utter AC, Nieman DC, Ward AN, et al. Use of the leg-to-leg bioelectrical impedance method in assessing body-composition change in obese women. Am J Clin Nutr 1999;69:603-7.

19 Vasold KL, Parks AC, Phelan DML, et al. Reliability and validity of commercially available low-cost bioelectrical impedance analysis. Int J Sport Nutr Exerc Metab 2019;29:1-5.

20 Browning LM, Mugridge O, Dixon AK, et al. Measuring abdominal adipose tissue: comparison of simpler methods with MRI. Obes Facts 2011;4:9-15.

21 Scharfetter $\mathrm{H}$, Schlager T, Stollberger R, et al. Assessing abdominal fatness with local bioimpedance analysis: basics and experimental findings. Int J Obes Relat Metab Disord 2001;25:502-11.

22 Unno M, Furusyo N, Mukae H, et al. The utility of visceral fat level by bioelectrical impedance analysis in the screening of metabolic syndrome - the results of the Kyushu and Okinawa Population Study (KOPS). J Atheroscler Thromb 2012;19:462-70.

23 Ozhan $\mathrm{H}$, Alemdar R, Caglar O, et al. Performance of bioelectrica impedance analysis in the diagnosis of metabolic syndrome. J Investig Med 2012;60:587-91.

24 Lee M-M, Jebb SA, Oke J, et al. Reference values for skeletal muscle mass and fat mass measured by bioelectrical impedance in 390565 UK adults. J Cachexia Sarcopenia Muscle 2020;11:487-96.

25 Uemura K, Doi T, Tsutsumimoto K, et al. Predictivity of bioimpedance phase angle for incident disability in older adults. $J$ Cachexia Sarcopenia Muscle 2020;11:46-54.

26 Kyle UG, Bosaeus I, De Lorenzo AD, et al. Bioelectrical impedance analysis-part II: utilization in clinical practice. Clin Nutr 2004;23:1430-53. 